\title{
Animal models of polymicrobial pneumonia
}

This article was published in the following Dove Press journal:

Drug Design, Development and Therapy

26 June 2015

Number of times this article has been viewed

\author{
Sami Hraiech ${ }^{1,2}$ \\ Laurent Papazian ${ }^{1,2}$ \\ Jean-Marc Rolain' \\ Fabienne Bregeon ${ }^{1,3}$
}

'IHU Méditerranée Infection, URMITE CNRS IRD INSERM UMR 7278,

Marseille, France; ${ }^{2}$ Réanimation -

Détresses Respiratoires et Infections

Sévères, APHM, CHU Nord, Marseille,

France; ${ }^{3}$ Service d'Explorations

Fonctionnelles Respiratoires, APHM,

CHU Nord, Marseille, France
Correspondence: Jean-Marc Rolain IHU Méditerranée Infection, URMITE CNRS IRD INSERM UMR 7278, 27 Bd J Moulin, I3385 Marseille Cedex 05, France

Email jean-marc.rolain@univ-amu.fr
Abstract: Pneumonia is one of the leading causes of severe and occasionally life-threatening infections. The physiopathology of pneumonia has been extensively studied, providing information for the development of new treatments for this condition. In addition to in vitro research, animal models have been largely used in the field of pneumonia. Several models have been described and have provided a better understanding of pneumonia under different settings and with various pathogens. However, the concept of one pathogen leading to one infection has been challenged, and recent flu epidemics suggest that some pathogens exhibit highly virulent potential. Although "two hits" animal models have been used to study infectious diseases, few of these models have been described in pneumonia. Therefore the aims of this review were to provide an overview of the available literature in this field, to describe well-studied and uncommon pathogen associations, and to summarize the major insights obtained from this information.

Keywords: pneumonia, animal model, co-infections, polymicrobial, route of infection

\section{Introduction}

Pneumonia is responsible for frequent hospitalizations and remains one of the leading causes of mortality from infectious diseases worldwide, ${ }^{1}$ with a $20 \%-30 \%$ mortality rate. ${ }^{2,3}$ The emergence of antibiotic resistance among the pathogens responsible for nosocomial and community-acquired pneumonia presents a challenge to delivering adequate treatment. ${ }^{4}$ In recent years, new antibiotics have been developed; however, the emergence of multidrug-resistant strains persists. ${ }^{5}$ Extensive research is necessary to obtain a better understanding of the mechanisms underlying the emergence of resistant strains and decipher host-pathogen and pathogen-pathogen interactions, particularly when polymicrobial invasion is concerned.

The physiopathology of pneumonia is more complex than expected when considering microbial pathogenicity in respiratory airways. The common idea of a single pathogen being responsible for the development of the observed pneumonia is likely misleading. In some cases, multiple microorganisms share the responsibility for the disease, whereas in other cases, the infection is the direct reflection of a first pathogen, favoring the subsequent multiplication of another microorganism. This concept, socalled "super-infection", was proposed 30 years ago to explain "chronic" viral infections, such as HIV infections. ${ }^{6}$ More recently, super-infection has also been described for acute pneumonia, with viruses or fungi as the primary causative agent, followed by a bacterial superinfection. ${ }^{7}$ The primary example of superinfection concerns Staphylococcus aureus or Streptococcus pneumoniae pneumonia occurring after influenza virus infection. ${ }^{8-10}$ More importantly, Herpesviridae could favor the development of bacterial pneumonia. ${ }^{11-13}$ Recent studies using molecular biology have confirmed previous pathological findings of frequent polymicrobial pneumonia, particularly in ventilator-associated pneumonia (VAP). ${ }^{14}$ However, many questions remain: how do multiple pathogens interact? How are host-pathogen responses balanced? What is the 
precise pathogen-specific pathogenicity, and how should this condition be treated?

Animal models have been extensively used in the field of pneumonia research and have often provided insight into the pathophysiology of this infection. Several models of acute or chronic pneumonia due to a single-pathogen species have been described, ${ }^{15}$ but there are few descriptions of polymicrobial pneumonia models.

The aims of this review are to present the main animal models of polymicrobial pneumonia developed thus far, to assess the mechanisms or treatments of pneumonia commonly observed in humans, and to discuss some other research perspectives.

\section{Polymicrobial pneumonia models: technical aspects Ethical considerations regarding animal experimentation}

Before all, one must remind that all experimental studies conducted on animals must have beforehand been evaluated and approved by ethic committees which have expressed similar guidelines in Europe and the USA. These institutions are the warrant that the wellbeing of animals is respected throughout the experiments. In the European Union, the legislation concerning animal studies has been recently reformed to reinforce the controls. Ethic committees are consisting of scientists, physicians and veterinaries, experts in the field of animal studies but also non-scientist members such as sociologists and philosophers. Associations for the protection of animals are also associated to the reflection on the respect of animals in experimental procedures. The European legislation has highlighted the importance of some concepts such as the justification of the use of animals and the number of animals concerned, the particular protection of some species (especially primates), the conditions of transport, the improvement of the accommodation conditions, the measures to avoid pain and anxiety. The competences required for the experimenters have been specified and the control of the institutions in which experiments are conducted reinforced. The "three Rs" concept summarizes the key points of the European and US legislation about animal protection during animal experimentations. "Replacement" refers to methods that avoid using animals. "Refinement" refers to modifications of husbandry or experimental procedures to enhance animal wellbeing and minimize or eliminate pain and distress. "Reduction" involves strategies for obtaining comparable levels of information from the use of fewer animals or for maximizing the information obtained from a given number of animals (without increasing pain or distress) so that in the long run fewer animals are needed to acquire the same scientific information.

\section{The selection of an appropriate animal species}

In pneumonia models, mammalians are most often used because of the anatomical and physiological proximity of these animals with humans. As non-mammalian species, such as birds, are typically studied for veterinary or zoonotic purposes, these models will not be further discussed herein. Larger mammalian species, such as rabbits, dogs, pigs, baboons, etc, are typically preferred when extensive physiological cardiovascular monitoring and hemodynamic support are assessed (Figure 1). Specifically, non-human primates are the only animal species able to evaluate primate-specific infectious agents, such as HIV. Because of the feasibility of lung mechanics measurements, large animals are preferred in VAP models. Although baboons ${ }^{16}$ have been used, piglets are the currently and more frequently used models. In contrast, polymicrobial infections have primarily been assessed in rodents, particularly mice and rats. The small size and rapid reproductive rate of rodents are conducive to many of the practicalities of laboratory research. Inbred mice strains are typically used to study genetically identical cohorts, and these animals facilitate the use of genetic approaches to understand molecular mechanisms of a disease. Genetic engineering of mouse embryonic stem cells is currently available, and a wide variety of transgenic mice harboring loss-of-function, gain-of function, or reporter genes have been generated. For example, the engineering of cystic-fibrosis-like mouse models to study airway colonization or chronic pneumonia might be of particular interest even if one must keep in mind that mice models do not exactly reproduce human pathophysiology of cystic fibrosis (CF) lungs. ${ }^{17,18}$ Because mice have become a mainstay of biomedical research, the development of new mouse studies benefits from the vast amount of existing literature regarding murine host defenses and immunological responses and the many relevant and readily available resources, such as recombinant mouse proteins or antibodies against mouse proteins.

The species selection also impacts the inoculation route. For example, mice and rats do not have cough reflexes, which make the nasal route a particularly interesting infection route in this species. In contrast, guinea pigs have a marked cough reflex, which explains the need to use intra-tracheal or aerosol routes. 


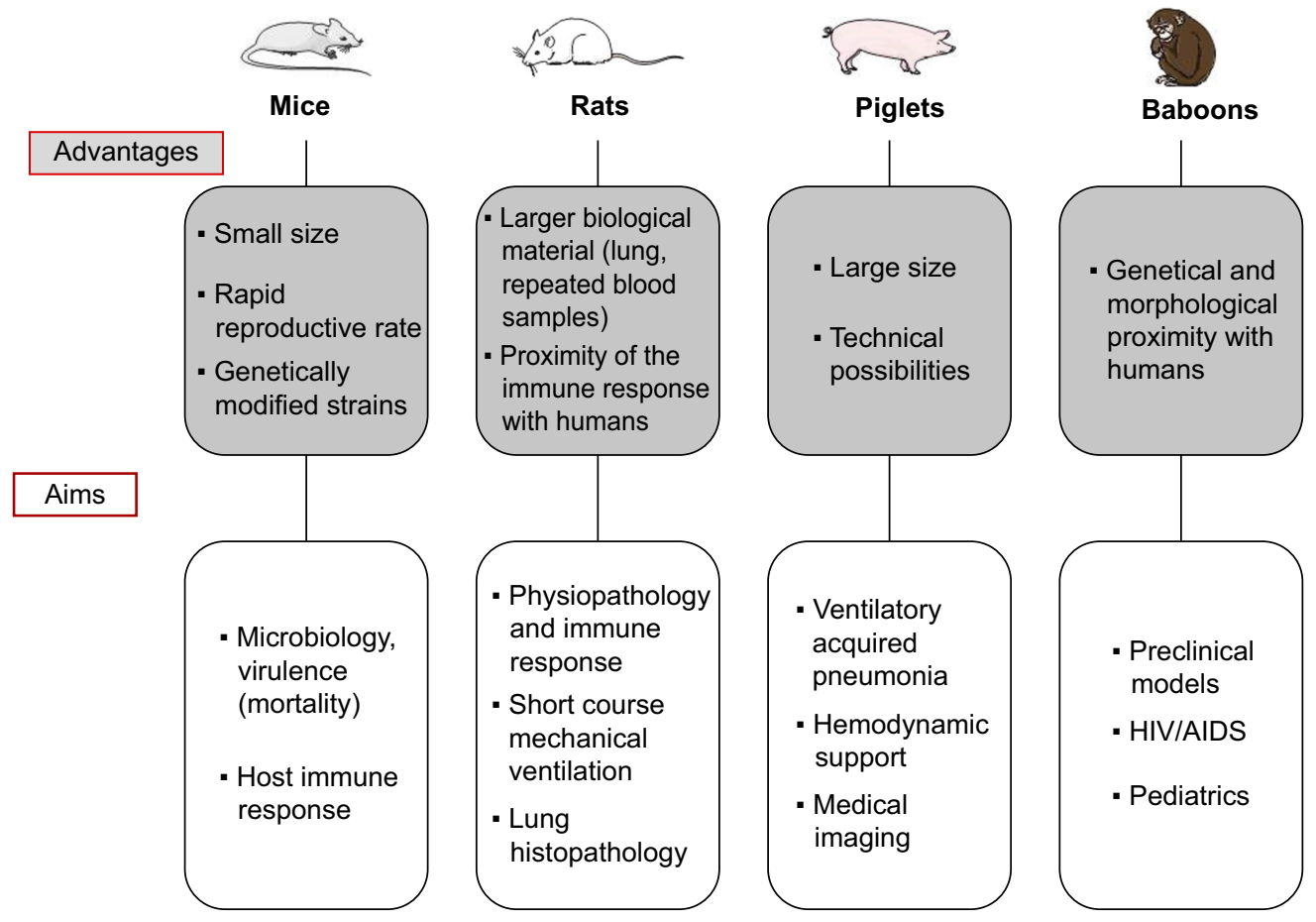

Figure I Characteristics and targets of frequently used species in animal models of pneumonia.

Notes: The main animal species used are presented. For each, the experimental advantages are mentioned in a panel followed by the list of the main topic for which these species are usually used.

\section{Inoculation routes}

Several inoculation routes have been described. Nasal inoculation is a simple method that can be used with several respiratory pathogens. ${ }^{19,20}$ For this technique, anesthetized mice are held in the vertical position, and the microbial suspension is deposited in the nares. The inoculum is aspirated into the lungs. Animals can be anesthetized for a short duration for this procedure, which is likely preferable to minimize variations in the rate and depth of respirations. Inoculation volumes vary from 5 to $50 \mu \mathrm{L}$. Smaller volumes typically remain localized in the upper respiratory tract, whereas higher volumes expose the animal to drowning without improving the efficacy of infection. ${ }^{21}$ This technique has particular relevance for modeling respiratory infections involving both the upper and lower respiratory tracts. However, the major limitation of intranasal instillation is the highly variable deposition of microorganisms in the lungs, with inter-animal differences in microbial deposition..$^{22}$ Sources of variability have primarily been associated with the expulsion of inoculation volume from the nose and the diversion of the inoculum into the alimentary tract.

Direct endotracheal inoculation is another route facilitating the direct deposition of the microorganisms into the respiratory tract, decreasing the risks of expulsion or esophageal deposition of the inoculum. This technique can be achieved using orotracheal intubation or tracheotomy, which includes a surgical procedure with potential complications. Both techniques necessitate the use of general anesthesia. Direct endotracheal instillation facilitates the inoculation of the microorganisms into a localized zone of the lungs. However, pneumonia concerns most often lower lung zones in a non-uniform manner. ${ }^{15}$

Many lower respiratory tract infections are acquired through the inhalation of infectious aerosols, including tuberculosis, legionellosis, mycoplasma and chlamydia infections, fungal pneumonias, and respiratory viral infections. Airborne transmission can be mimicked through the exposure of mice to aerosolized microorganisms in wholebody (Figure 2) or nose-only chambers. The inhalation of aerosolized microorganisms results in symmetrical deposition throughout both lungs. ${ }^{23}$ The disadvantages of aerosol models of infection include the costly equipment that must be regularly maintained and tested and the potential exposure of laboratory personnel to airborne biohazards. The deposition of aerosolized organisms in the upper respiratory tract, on the eyes and pelt, and clearance to the alimentary tract might be a confounding factor in some infections. Furthermore, some respiratory pathogens, such as $S$. pneumoniae or several respiratory viruses, are particularly sensitive to desiccation and/or oxygen toxicity and survive poorly when aerosolized. 


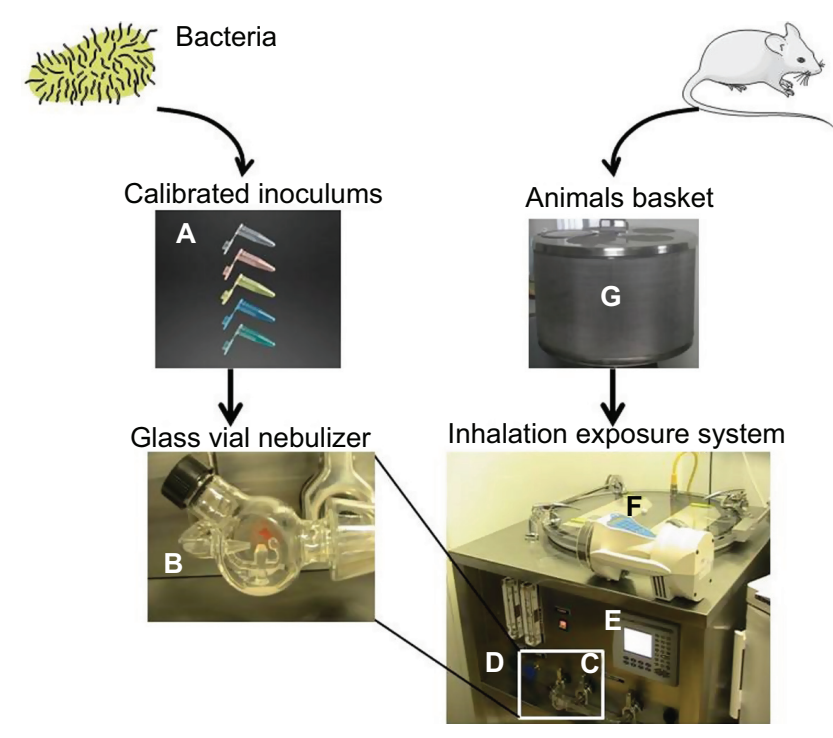

Figure 2 Whole-body inhalation exposure system.

Notes: The Glas-Col ${ }^{\circledR}$ aerosol exposure chamber system (Glas-Col, Terre Haute, IN, USA) is presented here. The bacterial suspension at a known concentration (A) is placed into a glass vial that is a Venturi nebulizer (B), connected to a leak-proof gas flow system (C). Pumps generate compressed gas flow to disperse the suspension into a fine mist. Pumps' flow rate is adjusted on manual flowmeters (D). Durations of the aerosolization and decontamination time are set via digital interface software displayed on a screen window (E). High-efficiency particulate arrestance filters, gas incinerators, and UV decontamination $(\mathbf{F})$ processes are all included in a whole block. The system operates under negative pressure. Before aerosolization starts, animals are placed in a stainless steel basket $(\mathbf{G})$.

Moreover, there is an inoculum upper limit achieved through inhalation, and higher dose infections can be achieved through bolus methods (nasal/intra-tracheal route).

\section{Acute or chronic infection}

Acute pneumonia models are preferred in mortality studies. Infection symptoms are rich and appear rapidly (within a few hours). Bacterial burdens in lungs are high during the first day of infection but rapidly decrease as the animal recovers. Conversely, when using chronic models, the animals exhibit less evident signs of sepsis, but the bacterial pulmonary count and histological lesions of bronchopneumonia remain present for a longer period, occasionally lasting for several weeks. ${ }^{24}$ The use of a vector solution containing the infectious agent is crucial for the kinetics of the spread of the microorganisms in the airways. Buffered suspensions, such as phosphate-buffered saline, or aqueous solutions facilitate rapid spread and favor the development of acute pneumonia. When chronic carriage is targeted, bacteria embedded in agar beads should be preferred (Figures 3 and 4). ${ }^{25}$ Beads mediate the slow dissemination of bacteria in the lungs and provide relative protection against immune cells; thus, bacteria persist in the respiratory tractus for several days. Alginate beads have also been successfully used with mucoid strains, as these materials favor biofilm development. ${ }^{26}$

\section{Time interval between successive inoculations}

In polymicrobial pneumonia models, the sequence of microorganism administration varies and is a key factor in the severity of infections. Generally, pathogens are successively administered because simultaneous inoculation is less relevant from a pathophysiological point of view. The time between the two infections varies from approximately 1 to 7 days, and even 21 days in some studies, and this time interval represents a determining factor in the synergism of pathogens. ${ }^{27}$ Concerning the associations between viruses and bacteria, the administration of bacteria prior to virus administration does not result in increased lethality, whereas viral infection increases bacterial virulence, with a climax when bacterial inoculation occurs 7 days after viral infection. A similar design has been observed in fungal-bacterial infections. However, in models of polybacterial pneumonia, mixed inoculums containing several pathogens are inoculated together. Indeed, the question of bacterial interaction and communication and the influence on biofilm formation is subtended in these models.

\section{Histological lesions}

From a histopathological point of view, the pulmonary alterations of mice infected with virus and secondarily with bacteria are more extensive than those of mice infected with either virus or bacteria alone. The extensive and severe consolidation of affected lobes and the obliteration of alveolar architecture were striking findings. Inflammatory infiltrates, epithelial cell hypertrophy and hyperplasia, and fibrin deposition were also common.

\section{Overview of available models of polymicrobial associations and pathophysiological contribution Association of viruses and bacteria Influenza virus models}

Bacterial superinfection is a common contributor to the severe outcome of influenza pneumonia. In the 19th century, the French physician Laennec documented the first viral-bacterial superinfections, noting the increased incidence of typical lobar pneumonia in patients suffering from influenza. This association was also described during the three influenza pandemics of the 20th century, with bacterial identification in $50 \%-95 \%$ of the patients with fatal or life-threatening influenza pneumonia. ${ }^{28}$ Moreover, bacterial superinfections were described during the recent 2009 H1N1 pandemic, where bacterial superinfections were observed in 

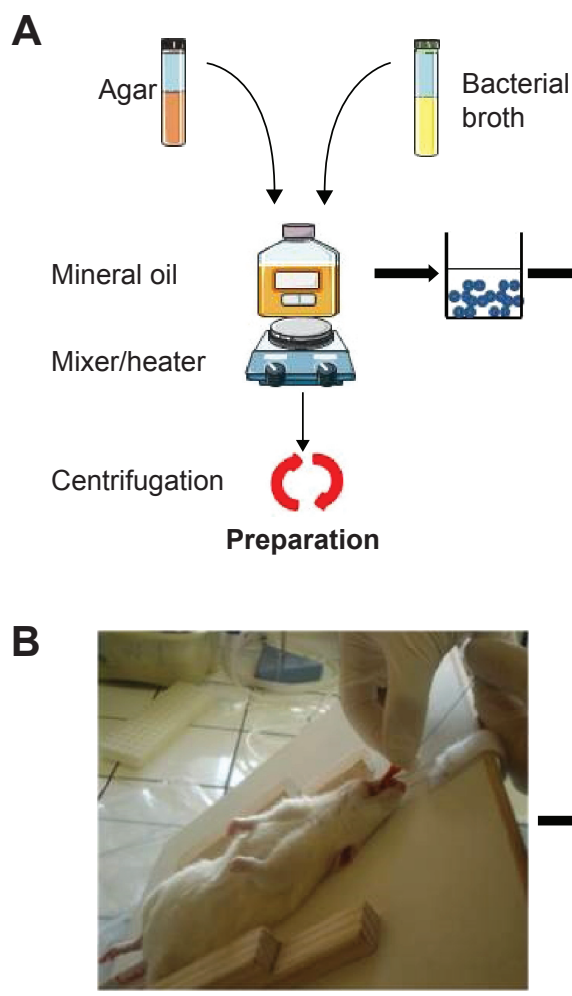

Rat tracheal intubation

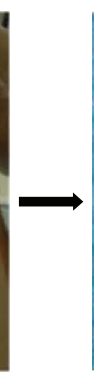

(1)

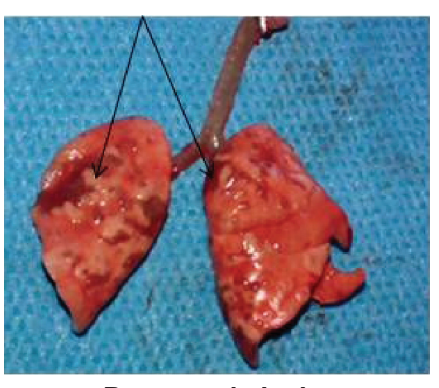

Pneumonia lesions

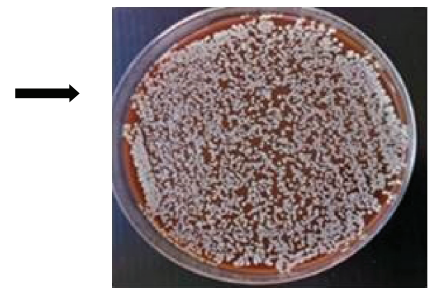

Control of infective inoculum
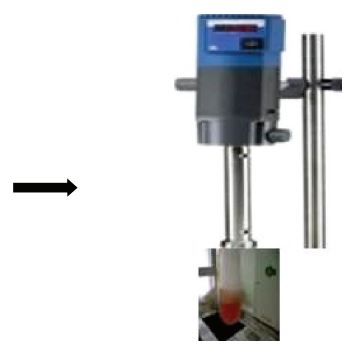

Lung homogenate for bacterial culture

Figure 3 Chronic pneumonia model using agar beads: main steps.

Notes: (A) Agar beads synthesis (top row of pictures). Broth containing bacteria and agar is added to mineral oil with continuous shaking and heating. The solution obtained is centrifuged to obtain beads whose size is measured and must be about $100 \mu \mathrm{M}$. The precise inoculum is assessed by serial dilutions method. (B) Model of rat pneumonia (bottom row of pictures). After inhaled anesthesia, the rat is suspended by the teeth and intubated into the trachea. Agar beads solution is injected into a tracheal catheter. After animal sacrifice, macroscopic aspects of lung lesions can be observed. Then, the lungs are homogenized for bacterial count.

$25 \%-56 \%$ of severe or fatal cases. ${ }^{8}$ Since the early $1930 \mathrm{~s},{ }^{29}$ mammalian models have been developed to explore influenza virus pathogenesis, among which swine, mice, ferrets, and guinea pigs are the most relevant and currently used models as described below.

\section{Animal models of influenza-S. pneumoniae superinfections}

In mice, the nasal inoculation of H1N1 influenza virus, followed 2 days later by an $S$. pneumoniae nasal inoculation has been associated with an increased mortality rate compared with single infections with either influenza virus or $S$. pneumoniae. ${ }^{30}$ In this study, although the viral titers did not vary between groups, the bacterial count was 10-100-fold higher in superinfected than in single-infected mice. Consistently, McCullers and $\operatorname{Regh}^{27}$ demonstrated lethal synergism between $S$. pneumoniae and influenza virus in mice, with $15 \%$ mortality with pneumococcal infection alone, 35\% mortality with influenza infection alone and $100 \%$ mortality with an experimental $S$. pneumoniae superinfection performed at 7 days after the first hit.
Mechanistic explanations have also been inferred from the above-mentioned studies: lung neutrophil function is altered and lung bacterial clearance is decreased when influenza virus infection precedes streptococcal infection. ${ }^{31}$ Moreover, the concentrations of inflammatory cytokines/ chemokines, including TNF- $\alpha$, IL-6, MIP-2, and RANTES, are higher in the lungs of co-infected mice than in the lungs of mice singly infected with either pathogen. An increase in cytokine release from $\mathrm{T}$ helper 1 cells has also been demonstrated in superinfection models, but no significant changes in cytokine production in T helper 2 cells were observed. ${ }^{30}$ Th1 cytokine activation exerts protective and antiviral effects, whereas Th2 cytokines exhibit inhibitory effects on viral clearance. The balance between Th1 and Th 2 cytokine activation might therefore represent a key mechanism in the pathogenesis of severe lung damage following influenzaS. pneumoniae superinfections. TLRs and associated MAPK signaling might also be involved. ${ }^{30} \mathrm{PAF}$ and its receptor PAFr were investigated in another mouse model, showing an upregulation in PAFr expression during the lung inflammatory response, facilitating bacterial invasion to cause bacteremia. ${ }^{32}$ 

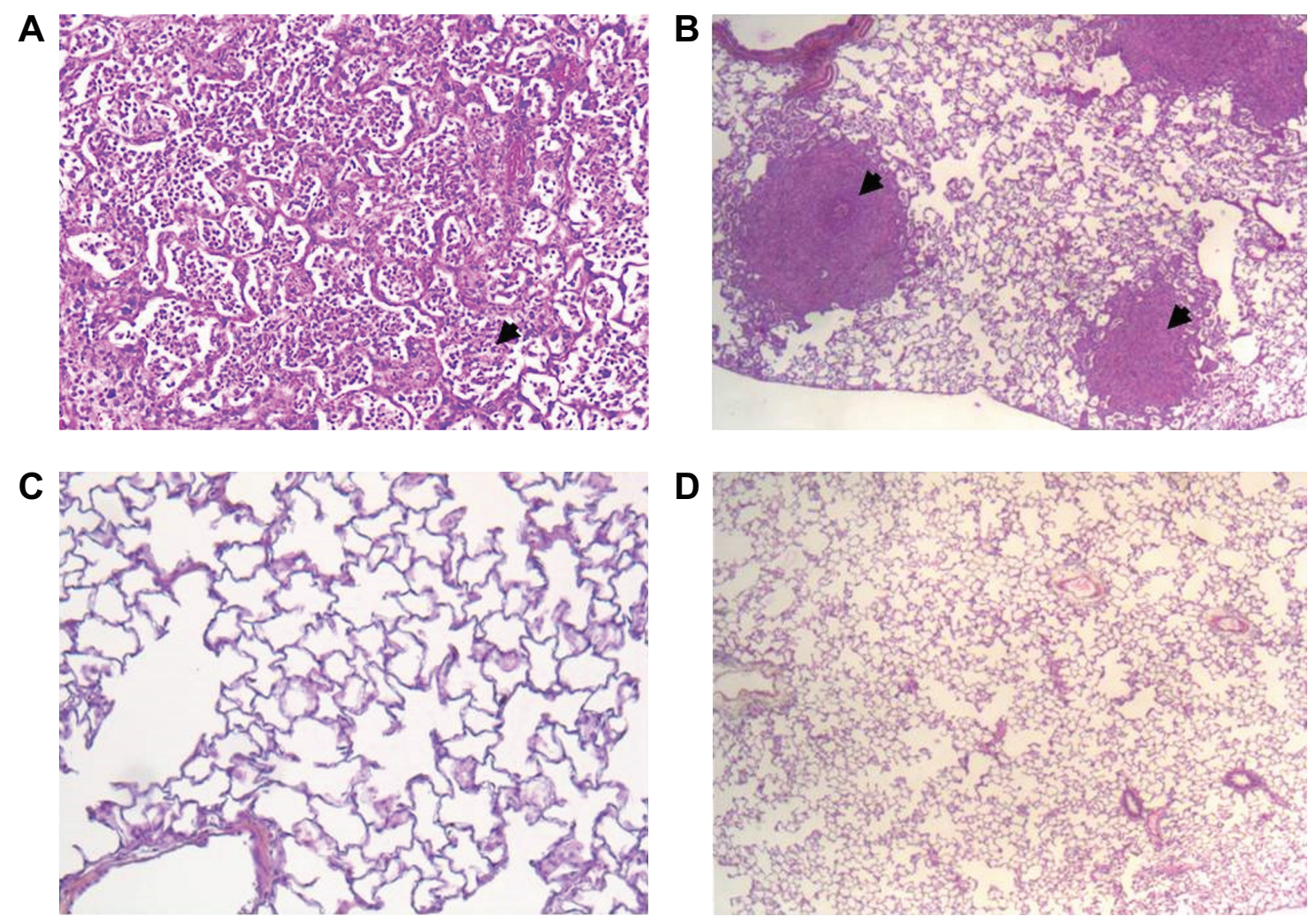

Figure 4 Histological pictures.

Notes: Magnification $\times 30$ showing lung lesions of acute $(\mathbf{A})$ and chronic Pseudomonas aeruginosa pneumonia. Chronic pneumonia was obtained with infected agar beads intra-tracheal inoculation (B). Black arrows indicate the lesions of bronchopneumonia. (C) Shows normal lung and (D) is issued from rat lungs inoculated with sterile agar beads and shows normal lung parenchyma.

The inflammatory activation of PAFr is an important factor in the attachment and invasion of cells by virulent pneumococcal strains bearing the natural PAF ligand, phosphorylcholine, on the cell surface..$^{33}$ Interestingly the inhibition of the PAF receptor delays the fatal outcome. ${ }^{32}$

Kosai et al also investigated the immunological mechanisms underlying the virulence of $S$. pneumoniae-influenza virus co-infection using a fulminant pneumonia mouse model. ${ }^{34}$ These authors observed a synergistic effect of influenza-S. pneumonia superinfections on the expression of A1AT and the production of neutrophil proteolytic enzymes, such as NE, MPO, and LYZ.

\section{Animal models of influenza-Staphylococcus superinfections}

Associations between influenza and S. aureus, particularly necrotizing pneumonia, have been rarely described in humans. ${ }^{35}$ Some animal models have been used to investigate this association. Using a model similar to that used for the nasal inoculation of both influenza and $S$. aureus, Lee et al showed that viral infection performed at 72 hours prior to bacterial infection accelerated death. ${ }^{36}$ Interestingly, and in contrast to the evidence discussed in the preceding section, the lung bacterial count in co-infected mice was not higher than in singly infected mice. However, superinfection increased the systemic dissemination of $S$. aureus (blood, spleen, kidneys, and liver). Infected mice showed altered immune cellular responses and high levels of IFN-gamma. ${ }^{36}$

In cotton rats co-infected with both infectious agents, including a clinical nosocomial methicillin-resistant S. aureus (MRSA) strain, the synergy of influenza and S. aureus exhibited a higher lethality, with higher bacterial lung counts and an increased incidence of bacteremia. Higher levels of the pro-inflammatory cytokines IL- $1 \beta$ and IL- 6 and anti-inflammatory cytokines, such as IL-10, were retrieved from the lungs of co-infected cotton rats. IFN-gamma levels were also increased. ${ }^{37}$

\section{Animal models of influenza and Haemophilus influenzae superinfections}

H. influenzae is one of the pathogens involved in bacterial pneumonia following influenza virus infection in humans. In a recent mouse model of dual infection, Lee et $\mathrm{al}^{38}$ reported a synergy between the two pathogens, independent of the delay 
between the inoculations (no lethality when administered together and high lethality when bacterial infection occurred 3 days after viral inoculation). As previously shown for influenza-S. pneumoniae superinfections, the authors observed that with influenza and $H$. influenzae, the time sequence of the inoculation was crucial. To achieve the highest pathogenicity, the virus should be infected prior to the bacteria, and reversion of the order of inoculation resulted in less pathological effects. This observation suggests the influence of the virus on the host ability to respond to bacterial infection.

\section{Influenza virus in non-rodent models}

A major disadvantage of mice models of influenza is that mice are not a natural host for human influenza A infection. Ferrets and pigs are useful models for studying lower respiratory tract flu infections (with non-avian strains). The attachment of the virus to the cells in ferrets and pigs is similar to that observed in humans. Using a pig model, Loving et $\mathrm{al}^{39}$ showed that flu infection decreases the clearance of Bordetella bronchiseptica, a bacteria that causes pulmonary disease in animals and immunocompromised humans (such as $\mathrm{CF}$ patients). ${ }^{40} \mathrm{Co}$-infection is responsible for the enhanced production of pro-inflammatory mediators that likely contribute to exacerbated pulmonary lesions.

\section{Infections following metapneumovirus pneumonia}

Human metapneumovirus (hMPV) is a recently described paramyxovirus that causes respiratory tract infections. Considering the evidence of bacterial superinfections following influenza pneumonia, Kukavica-Ibrulj et $\mathrm{al}^{41}$ investigated the effects of a primary viral infection with hMPV or influenza A virus followed by $S$. pneumoniae superinfection 5 days later. For the same infecting bacterial inoculum, both groups of superinfected mice exhibited a more severe pneumonia than animals infected with bacteria alone. Bacterial counts increased from $5 \times 10^{2} \mathrm{CFU} / \mathrm{lung}$ in mice infected with pneumococcus to $10^{7}$ and $10^{9} \mathrm{CFU} /$ lung in mice previously infected with hMPV and influenza A virus, respectively. Marked interstitial and alveolar inflammation was also observed. Increased damage associated with higher levels of inflammatory cytokines and chemokines, such as IL-1, IL-6, IL-12, MCP1, MIP-1, the mouse IL-8 homolog $\mathrm{KC}$, and GCSF. The increased expression of TLRs TLR2, TLR6, TLR7, and TLR13 was also observed in the lungs of superinfected animals compared with singly infected models. Regardless of the priming virus, hMPV or influenza A, similar immunological effects were observed.
Bacterial superinfection of viral pneumonia: what do animal models convey?

Animal models have provided numerous data and many insights in the field of viral and bacterial pneumonia: first, these data confirmed the pathogen synergism of co-infection; second, altogether, the results suggest that viral infection primes the host for bacterial pneumonia, making the subject prone to subsequently develop a more severe form of infection; and third, these data suggest that the role of the immune response is crucial, including an enhanced inflammatory response likely responsible for increased alveolar damage. In addition, global "immunosuppression" might favor the impaired clearance of microorganism from airways. Moreover, adhesion molecules have also been implicated in the severity of bacterial pneumonia (Figure 5).

More viruses than initially expected "open the road" to bacteria, and in some cases of immunosuppression, the virulence of weakly pathogenic bacteria could be increased. Given that viruses from various origins share some common physiopathological mechanisms of lung pathogenesis, it is reasonable to consider the potential priming effect of numerous other viruses, particularly those exhibiting respiratory tropism. A specific concern in mechanically ventilated patients is the reactivation of Herpesviridae associated with the genesis of bacterial pneumonia. ${ }^{10,11}$ To better assess this issue, animal models of bacterial pneumonia following Herpesviridae infections are required.

\section{Multiple bacteria pneumonia: a microbiological conspiracy? Clinical background}

With the recent development of molecular biology and mass spectrometry for the identification of microorganisms in pulmonary samples obtained from patients with pneumonia, microbiologists and clinicians have observed polymicrobial infections. Molecular biology experiments performed on bronchoalveolar lavages obtained from intensive care unit (ICU) patients showed that $30 \%$ of bronchoalveolar lavages were polymicrobial, particularly samples obtained from patients with VAP. ${ }^{14}$ Other studies have shown similar results in samples from patients exhibiting community-acquired pneumonia. ${ }^{42}$ These results lead to questions concerning the responsibility of the isolated pathogens and suggest that the association of several bacteria, rather than a sole pathogen, results in the observed clinical symptoms. Animal models supporting this idea would provide interesting insights, but these data are surprisingly scarce. Most polymicrobial models involve secondary pneumonia after the initial triggering of 


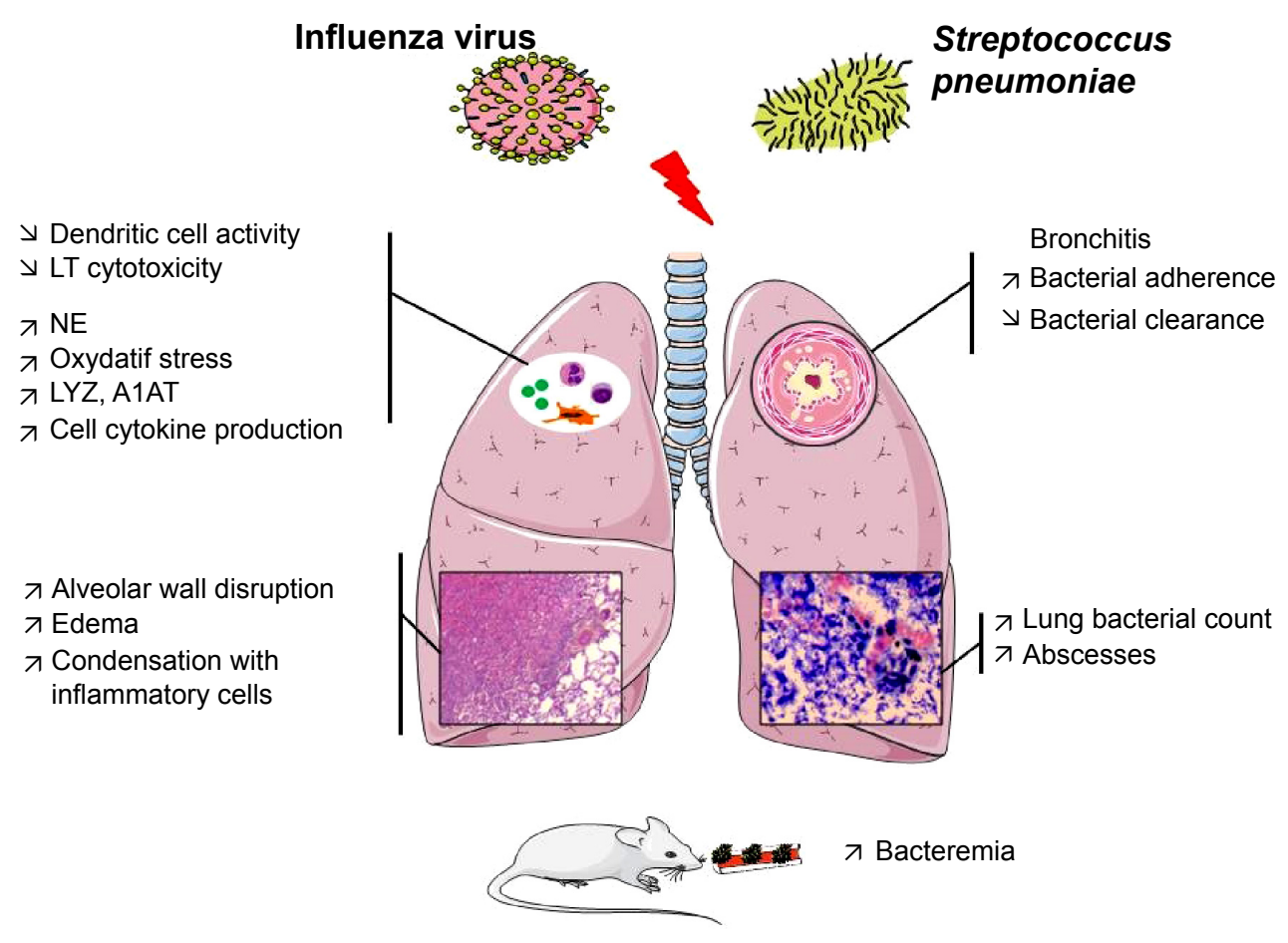

Figure 5 Pathophysiological mechanisms explaining the severity of a Streptococcus pneumoniae pneumonia following an influenza virus lung infection according to mice models. Notes: When influenza pneumonia is followed by a streptococcal superinfection, the severity resulting from the synergy of both microorganisms can be observed at different levels. In the lungs, it is associated with edema, inflammation, and impaired immune response. From a microbiological point of view, the bacterial clearance is decreased and lung abscesses are more frequently described. Extra-pulmonary bacterial dissemination is also more favored with an increased occurrence of bacteremia.

Abbreviation: AIAT, alphal antitrypsine; LYZ, lysozyme; NE, neutrophil elastase; LT, T lymphocytes.

extrapulmonary sepsis in rat models of cecal ligature and punction. ${ }^{43}$ Excluding models of pneumonia with polymicrobial abscesses and anaerobes, the majority of aerobic polybacterial pneumonia models concern CF.

\section{CF models of polybacterial pneumonia}

\section{Pseudomonas aeruginosa with S. aureus}

$S$. aureus most commonly colonizes the respiratory tract of CF patients, typically during childhood. P. aeruginosa progressively becomes the predominant pathogen in adolescents and young adults. These two pathogens co-exist in CF patients, and the interaction between these microorganisms likely influences their pathological potential. ${ }^{44}$ In a recent in vitro study, Baldan et $\mathrm{al}^{45}$ showed that "early" clones of P. aeruginosa (ie, strains collected in patients at the early phase of the disease) outcompeted $S$. aureus growth and exhibited biofilm mass formation. This result was not observed using "late" clones of $P$. aeruginosa (with several patho-adaptive traits induced through multiple antibiotics treatments), as these clones show reduced virulence. These results were confirmed in a mouse model of dual pneumonia with $S$. aureus and $P$. aeruginosa (simultaneous co-infection). In the lungs of co-infected mice, early clones, but not late clones, of $P$. aeruginosa outcompeted $S$. aureus growth. It is plausible that late clones of $P$. aeruginosa are less competitive, reflecting the antibiotic-induced patho-adaptive and reduced virulence of these microbes. These data underlie the importance of bacterial interactions in lung infections and particularly in the complexity of the interactions between different pathogens that co-exist in the airways of CF patients.

\section{P. aeruginosa with Burkholderia cenocepacia}

$P$. aeruginosa and $B$. cenocepacia are opportunistic human pathogens responsible for severe nosocomial infections in $\mathrm{CF}$ and immunocompromised patients. These two bacteria form biofilms in the airways of CF patients, making these infections more difficult to treat. Scientists have recently begun to appreciate the complicated interplay between microorganisms during polymicrobial infections of the $\mathrm{CF}$ airway $^{46}$ and the potential implications of this activity in disease prognosis and responses to therapy. To obtain insight into the potential relevance of the interactions between strains of $P$. aeruginosa and B. cenocepacia during infection, Bragonzi et al ${ }^{47}$ characterized co-inoculations using both in vitro and in vivo models. Co-inoculations were examined in an in vitro biofilm model. Thereafter, these authors infected cystic fibrosis transmembrane regulators-deficient mice with $P$. aeruginosa and B. cenocepacia strains embedded in agar beads at a 1:10 ratio via tracheal administration, resulting in a chronic lung co-infection. The data showed 
that $B$. cenocepacia positively influenced $P$. aeruginosa biofilm development by increasing biomass formation. Interestingly, mouse models of co-infection revealed that $P$. aeruginosa retained the ability to establish chronic infection in the presence of $B$. cenocepacia, but co-infection increased biofilm formation and the host inflammatory response. These results indicate that co-infection with $P$. aeruginosa and B. cenocepacia might enhance biofilm formation and the host inflammatory response. Therefore, alterations in bacterial behavior due to interspecies interactions might be important for disease progression and persistent infection.

The importance of bacterial biofilm formation has also been demonstrated in clinical devices, such as endotracheal tubes, in ICU patients. ${ }^{48}$ Biofilm formation is one of the mechanisms through which the endotracheal tube facilitates bacterial contamination of the lower airways. A higher number of bacterial species is obtained using molecular biology and pyrosequencing than using traditional culture methods. As it is highly likely that biofilm formation plays a crucial role in the development of polybacterial infection, the development of clinically relevant animal models of polybacterial infections would be of great interest.

\section{Anaerobic lung abscess models}

In a rabbit model, the intra-tracheal inoculation of anaerobes obtained from the dental flora of healthy humans, containing Gemella, Fusobacterium nucleatum, Eubacterium lentum, Bacteroides fragilis, and group C Streptococcus, resulted in the development of multiple lung abscesses and empyema after 12 days, with the same bacteria isolated in culture. ${ }^{49}$ This slightly lethal model is bacteriologically well defined and is currently one of the only available polymicrobial anaerobic pneumonia models. Recently, lung abscesses were developed in mice after inoculation with anaerobic bacteria (Porphyromonas gingivalis and Treponema denticola) obtained from patients with periodontal disease. ${ }^{50}$ Similar to the results obtained from bacterial superinfection during viral pneumonia, mixed infection has been associated with the delayed clearance of bacteria and increased expression of inflammatory cytokines, such as TNF $\alpha$, IL1 $\beta$, and IL6. These models underlie the pathogenic potential of anaerobes from the oral sphere to induce the development of lung abscesses.

\section{Associations with fungi Candida albicans with P. aeruginosa}

The role of fungal pathogens is unclear in immunocompetent hosts. ${ }^{2}$ Candida species are detected in over $50 \%$ of ventilated critically ill patients, ${ }^{7}$ but this microorganism is uncommonly responsible for lung infections. ${ }^{51,52}$ Thus, the existence of true Candida pneumonia in critically ill patients remains unknown..$^{53}$ In contrast, an association between Candida species airway colonization and subsequent $P$. aeruginosa VAP has been demonstrated, ${ }^{54}$ suggesting that interactions between bacterial and fungal species could be more important than previously considered. In a recent study, ${ }^{55}$ Roux et al developed an intra-tracheal C. albicans instillation model in Wistar rats, and evaluated the inflammatory response induced after C. albicans colonization based on high IFN-gamma concentrations. Interestingly, fungal airway colonization increased the development of $P$. aeruginosa pneumonia. IFN-gamma inhibited the phagocytosis of unopsonized bacteria by alveolar macrophages. Colonization was decreased in animals treated with antifungal drugs, and these animals were less susceptible to $P$. aeruginosa infection. These results were consistent with the results obtained from studies in rat models ${ }^{56}$ showing that Candida airway colonization increased the severity of $P$. aeruginosa pneumonia through the impairment of macrophage function. The reaction of the host immune system to the presence of the nonpathogenic Candida could reduce the clearance of pathogenic bacteria. Different conclusions have been drawn from another mouse study, ${ }^{57}$ in which the "protective effect" of $C$. albicans airway colonization was associated with increased $P$. aeruginosa clearance. This discrepancy between experiments might reflect a difference in the immune response developed in mice compared with rats during Candida colonization, implicating a Th2 response. Roux et $\mathrm{al}^{56}$ observed a specific Th1-Th17 response, the same immune response that protects against candidiasis in humans. ${ }^{58}$

\section{Cytomegalovirus with Pneumocystis jiroveci}

Using a mouse model of intraperitoneal murine cytomegalovirus infection and the simultaneous intra-tracheal infection with $P$. jiroveci, Qureshi et $\mathrm{al}^{59}$ explored the pathogenicity of these microorganisms in pneumonia in immunocompromised patients. Co-infected BALB/c mice exhibited a severe form of Pneumocystis infection, with increased weight loss and Pneumocystis burden in the lungs and a decrease in the clearance of this pathogen. From an immunological point of view, the authors interestingly demonstrated that murine cytomegalovirus infection was accompanied by down-regulatory effects on dendritic cells, as indicated by reduced numbers of MHC-II- and CD40-expressing cells during the early phase of infection. This effect was concomitant with a diminished CD4 cellular response and delayed pneumocystis clearance from the lungs of dual-infected mice compared with singly infected mice. Thus, this model suggests that concomitant cytomegalovirus and pneumocystis infections result in a virus-mediated altered immune response to pneumocystis 
with a prolonged course of pneumocystis pneumonia and more severe clinical illness.

\section{Parasites}

\section{Trypanosoma cruzi with P. jiroveci}

Few models have addressed the interactions between parasites and other microorganisms during pneumonia. One model was used to investigate the association between $T$. cruzi and $P$. jiroveci in rats and mice. ${ }^{60}$ The association between T. cruzi intraperitoneal infection and dexamethasone treatment was significantly associated with lethal secondary $P$. jirovec $i$ pneumonia, suggesting that Chagas disease patients treated with corticosteroids might be at risk for $P$. jiroveci pneumonia and should be considered for chemoprophylaxis.

\section{Discussion}

Herein, we reviewed animal models of polymicrobial pneumonia to describe the elements of pathophysiological mechanisms and their potential clinical significance (Table 1).
Whereas many animal models are available for single-pathogen pneumonia, ${ }^{13}$ polymicrobial designs have been poorly developed. This lack might reflect the complexity of these models requiring a host adapted to several micro-organisms, adequate timing between inoculations with different pathogens, and the determination of an effective inoculum that is not too lethal.

Animal studies represent approximately 3\% of the totality of studies published to date in the field of pneumonia as indexed in PubMed browser. However, constant growth of published articles using animal models in the last 10 years is in contrast with their scientific limits with regard to their reproducibility in humans. ${ }^{61}$ Technical constraints associated with the accommodation of animals and the recently reinforced legislation related to their wellbeing have come to superimpose on the infectious risk for animal experimentation manipulators, especially in the field of infectious agents with airborne transmission responsible for pneumonia. What would be the "ideal model" of pneumonia should be questioned first. At least, the chosen model should use strains

Table I Characteristics of the major pathogen associations described in animal models of pneumonia

\begin{tabular}{|c|c|c|c|c|}
\hline Pathogens & Host & Clinical/ microbiological effects & Immune response & Reference \\
\hline \multicolumn{5}{|l|}{ Viruses-bacteria } \\
\hline \multirow[t]{9}{*}{ Influenza-Streptococcus pneumoniae } & Mice & $\lambda$ mortality & Alteration of neutrophils' function & 31 \\
\hline & & $\pi$ lung bacterial count & & \\
\hline & & $\searrow$ bacterial clearance & & \\
\hline & & $\pi$ mortality & $\pi$ TNF $\alpha$, IL6, MIP-2, RANTES & 30 \\
\hline & & $\lambda$ lung bacterial count & $\pi \mathrm{T}$ helper cytokines & \\
\hline & & $\lambda$ mortality & $\pi$ TLR expression & \\
\hline & & $\lambda$ delay of mortality by PAFr inhibition & $\pi$ PAFr expression & 27 \\
\hline & & $\pi$ mortality & $\lambda$ AIAT, NE, MPO, LYZ & 34 \\
\hline & & $\lambda$ lung damage & $\rightarrow$ alteration of neutrophils' function & \\
\hline \multirow[t]{5}{*}{ Influenza-Staphylococcus aureus } & Mice & $\lambda$ bacteremia, liver, kidney abscesses & $\lambda I F N-g a m m a$ & 36 \\
\hline & & & $\checkmark$ cellular response & \\
\hline & Cotton & $\lambda$ mortality & ス ILI $\beta$, IL6, ILI0, IFN-gamma & 37 \\
\hline & rats & $\lambda$ lung bacterial count & & \\
\hline & & $\lambda$ bacteremia & & \\
\hline Influenza-Haemophilus & Mice & $\lambda$ mortality & $\searrow$ Native immune response & 38 \\
\hline \multirow[t]{4}{*}{ Metapneumovirus-S. pneumoniae } & Mice & $\pi$ lung bacterial count & $\pi$ ILI, IL6, ILI2 & 41 \\
\hline & & $\pi$ alveolar/interstitial inflammation & $\pi \mathrm{MCP}$ & \\
\hline & & & $\pi \mathrm{MIP}$ & \\
\hline & & & オ TLR2, TLR6, TLR7, TLRI3 & \\
\hline \multicolumn{5}{|l|}{ Bacteria-bacteria } \\
\hline Pseudomonas aeruginosa-S. aureus & Mice & $\searrow$ S. aureus biofilm and lung growth & Not evaluated & 45 \\
\hline Burkholderia cenocepacia-P. aeruginosa & Mice & $\lambda$ biofilm formation of $P$. aeruginosa & $\pi$ Inflammatory response & 47 \\
\hline \multicolumn{5}{|l|}{ Fungi/bacteria } \\
\hline \multirow[t]{4}{*}{ Candida albicans-P. aeruginosa } & Rats & $\lambda$ lung bacterial count & $\pi$ IFN-gamma & 55 \\
\hline & & & $\searrow$ phagocytosis & \\
\hline & Rats & $\lambda$ severity $P$. aeruginosa pneumonia & $\forall$ macrophage function & 56 \\
\hline & Mice & Protective effects against $P$. aeruginosa & $\lambda$ clearance of $P$. aeruginosa & 57 \\
\hline \multicolumn{5}{|l|}{ Viruses-fungi } \\
\hline \multirow[t]{2}{*}{ CMV-Pneumocystis jiroveci } & & $\pi$ weight loss & $\checkmark$ dendritic cells function & 59 \\
\hline & & $\searrow$ Pneumocystis clearance & $У$ CD40 response & \\
\hline
\end{tabular}

Abbreviation: CMV, cytomegalovirus. 
from environmental or clinical conditions, be inoculated through the airways, with an inoculum size plausible with the natural history of the target disease, in a host whose immunity and anatomy are as close as possible to those of the human. Regrettably, the choice of the final animal experiment design is often the result of a compromise between technical/financial imperatives for the researcher, the risk of infectious transmission, and the question asked. Indeed, the primate, close to humans' physiology, is legally reserved to specific topics, and the pig has important physiological similarities with humans but raises logistic and infectious problems for its maintenance and the volume of infective inoculums required. On the contrary, small animals, more easy to maintain and well known from a biological pointof-view, are however relatively far from humans' physiology and anatomy. Rats and mice are frequently naturally resistant to many pathogens so numerous transformations are frequently used to favor a susceptibility to infection by the mean of various ways of immunosuppression that takes us even further away from initial clinical context. Conversely, some animal species are more susceptible to pulmonary infections and should be preferred depending on the microorganism tested.

Expectedly, most models of polymicrobial pneumonia have been developed in rodents and particularly in mice. Their small sizes and rapid reproductive rates are conducive to many of the practicalities of laboratory research. Inbred strains mediate the study of genetically identical cohorts and facilitate the use of genetic approaches for understanding molecular mechanisms of disease. The genetic engineering of mouse embryonic stem cells is currently available to most investigators, and a wide variety of transgenic mice harboring loss-of-function, gain-of function, or reporter genes have been generated in cell specific or condition-specific manners. Because mice have become a mainstay of biomedical research, the development of new mice models might benefit from the vast amount of existing literature regarding murine host defenses and immunology for comparison. In addition, many relevant resources, such as recombinant mouse proteins or antibodies against mouse proteins, are readily available.

Regarding the multiple pathogen association studied, it appears that viral-bacterial associations are by far the most developed, and among them, influenza models, reflecting the clinical impact of these associations and particularly those described during historical flu epidemics. It permitted a better understanding of the pathophysiology of co-infections and particularly the role of host immune responses to identify future treatment targets.
One of the main conclusions obtained from the available polymicrobial pneumonia models is that these infections are not only associated with high morbidity but also with the synergy of both pathogens in lethality. The clinical effects (weight loss, behavior alteration, clinical sepsis score, mortality) of co-infections are often higher than the sum of the effects observed in single-pathogen infections. This observation strongly suggests the existence of a "missing link" between the pathogens, perhaps corresponding to the host immune response.

The role of the host immune response is crucial in the pathogenesis of polymicrobial pneumonia. Both cellular and humoral immune responses have been implicated. Several animal models of viral and bacterial pneumonia have shown increased levels of pro-inflammatory and antiinflammatory cytokines. Viral stimulus induces an immunosuppressive state that favors secondary bacterial infection, limits pathogen clearance, increases bacterial adhesion and bacteremia, and enhances bacterial virulence. This has been confirmed by the absence of increased virulence when the bacteria are administered prior to virus infection. Impaired neutrophil function reduces bacterial clearance in the lungs and diminishes the importance of adherence molecules, such as PAF. The role of T helper cell dysfunction has also been determined. ${ }^{17,18}$ Pathogen infections likely trigger an innate immune response in the host leading to a systemic inflammatory response, followed by immune dysfunction in favor of the emergence of secondary infections. Dendritic cells link innate and adaptive immunity and might be centrally involved in the regulation of sepsis-induced immune dysfunction. ${ }^{62}$

The airway epithelial mechanical barrier alteration may also be this "missing link" as it plays a crucial role in the penetration of microorganisms and host invasion. The airway epithelium lays at the interface between the external environment and the lung. It represents the first contact for microorganisms. The low permeability of the healthy pulmonary epithelium is in a large part due to tight junctions (TJ). TJ dysfunction induced by microorganisms may be involved in pathogen virulence and host invasion. In particular, $P$. aeruginosa elastase compromises the human airway epithelium and increases paracellular permeability in epithelial cell monolayers by mechanisms involving TJ disruption and cytoskeletal reorganization, leading to destruction of epithelial barrier function. ${ }^{63}$ In chronic pulmonary diseases such as $\mathrm{CF}$, impaired $\mathrm{TJ}$ function, as a consequence of chronic inflammation, is probably responsible for increased susceptibility toward microorganisms' invasion. ${ }^{64}$ Rodent models 
showed that $P$. aeruginosa lipopolysaccharide increased airway paracellular permeability and the number of opened TJ, a phenomenon dependent of MLCK. MLCK could therefore be a potential target for novel drugs intended for relief of lung injury. ${ }^{65}$ Whether alteration of TJ may play a role in polymicrobial pneumonia would need specific investigations. It is plausible that the observed synergy between pathogens into the infected lung relies on disruption of the airway barrier by the first pathogen favoring the invasion of the subsequent microorganism.

\section{Perspectives}

The inconvenience and limits of animal models of pneumonia are counterbalanced by the advances that they give in the comprehension of the interaction between hosts and pathogens and the development of future therapeutic ways.

\section{New researches and application progress of commonly used optical molecular imaging technology}

With the advent of new technologies such as intravital microscopy, the era of a "static" description of the behavior of the actors of anti-infectious defenses has let the place to a dynamic description with modified species harboring fluorochrome-marked cells. It is now possible to observe in real time the traffic of immunity cells and their interaction with the surrounding tissue and in particular the vascular endothelium. ${ }^{66}$ In the field of pneumonia research, it may perhaps allow describing in detail the development of severe lung lesions and the systemic dissemination of infection and may permit to identify new therapeutic targets. New advances in optical molecular imaging technology is also highly contributive to animal research. ${ }^{67}$

Various markers are available such as fluorescent protein, luciferase, fluorescent material, nanoparticle or others that can be used for further in vivo whole-body imaging. It allows the same animal to be its own control so animal numbers required for evaluation can be reduced. Therefore, inflammatory cells and/or bacteria can be tagged and infectioninduced inflammatory cells or bacterial infiltration of the lung or other organs could be dynamically imaged, allowing quantitative data to be collected in all organs simultaneously. Bioluminescence imaging has been recently used for polymicrobial tuberculosis challenge using distinct bioluminescent spectra and allowed co-localization of two different bacterial strains of Mycobacterium tuberculosis. ${ }^{68}$ Despite the fact that it could bring new insight into the in vivo behavior of co-infecting pathogens and their interaction with the host as well as important advances in pharmacological research to treat infections, to our knowledge, this technique has not yet been reported in polymicrobial models of pneumonia.

\section{New therapeutic ways}

Because physicians always have to face more challenging issues in the treatment of pneumonia, animal models remain essential and particularly valuable to evaluate new therapeutic ways. Indeed, the dramatic increase of antibiotic resistance in bacterial isolates from nosocomial pneumonia or patients with chronic lung infections leads to an important reduction of the therapeutic arsenal. ${ }^{69}$ However, the "pipeline" of new antibiotics developed is running dry ${ }^{5}$ and physicians are facing the threat of extremely-resistant strains. ${ }^{70}$ In vivo pneumonia models have recently permitted to evaluate future perspectives of new drugs to fight "super bugs". Gram-negative bacteria are of particular concern and recently, new therapeutic approaches have been tested with success in rodent models of $P$. aeruginosa pneumonia. In particular, the way of quorum sensing inhibition through the enzymatic hydrolysis of acyl-homoserine-lactones (with lactonases), implicated in the virulence and biofilm production of $P$. aeruginosa, is under extensive research. Animal models of acute pneumonia have shown promising results in this field. Recently, the demonstration of a reduction of mortality in rats pre-treated with a newly engineered inhaled lactonase was made. ${ }^{71}$ Similarly, the use of bacteriophages (viruses able to specifically "kill" bacteria) in the treatment of pneumonia implicating multidrug-resistant strains (especially in some specific populations such as CF patients) might be a promising alternative or adjunctive therapy combined with antibiotics. Again, the first results obtained in mice models of pneumonia using clinical strains from CF patients suggest an antibacterial efficacy with a reduction of mortality and a preventive effect against $P$. aeruginosa lethal pneumonia. ${ }^{72}$ One can expect that given the results obtained in animal models, the first clinical trials investigating these new therapeutics in pneumonia will soon begin, underlining the importance of experimental studies in the research on pulmonary infections.

\section{Conclusion}

In this review, we focused on the role of animal models in the comprehension of the pathogenicity of polymicrobial pneumonia. The main conclusion obtained from experimental models is that the high severity of the associations between microorganisms may be correlated with the immunomodulation induced by the first pathogen, particularly in viral infections but also with the alteration of the airway epithelial mechanical barrier. Because the currently available models 
are limited, the development of future models will likely help to define the role of pathogen interactions in pneumonia and contribute to the development of innovative drugs with new targets.

\section{Disclosure}

The authors have no conflicts of interest concerning this review.

\section{References}

1. Lopez AD, Mathers CD, Ezzati M, Jamison DT, Murray CJ. Global and regional burden of disease and risk factors, 2001: systematic analysis of population health data. Lancet. 2006;367(9524):1747-1757.

2. American Thoracic Society; Infectious Diseases Society of America. Guidelines for the management of adults with hospital-acquired, ventilator-associated, and healthcare-associated pneumonia. Am J Respir Crit Care Med. 2005;171(4):388-416.

3. Venditti M, Falcone M, Corrao S, Licata G, Serra P; Study Group of the Italian Society of Internal Medicine. Outcomes of patients hospitalized with community-acquired, health care-associated, and hospital-acquired pneumonia. Ann Intern Med. 2009;150(1):19-26.

4. Maragakis LL. Recognition and prevention of multidrug-resistant Gramnegative bacteria in the intensive care unit. Crit Care Med. 2010;38 (8 Suppl):S345-S351.

5. Bassetti M, Ginocchio F, Mikulska M. New treatment options against gram-negative organisms. Crit Care. 2011;15(2):215.

6. Hopewell PC. Prevention of lung infections associated with human immunodeficiency virus infection. Thorax. 1989;44(12):1038-1044.

7. Hamet M, Pavon A, Dalle F, et al. Candida spp. airway colonization could promote antibiotic-resistant bacteria selection in patients with suspected ventilator-associated pneumonia. Intensive Care Med. 2012; 38(8):1272-1279.

8. Glezen WP. Serious morbidity and mortality associated with influenza epidemics. Epidemiol Rev. 1982;4:25-44.

9. Mauad T, Hajjar LA, Callegari GD, et al. Lung pathology in fatal novel human influenza A (H1N1) infection. Am J Respir Crit Care Med. 2010;181(1):72-79.

10. Estenssoro E, Rios FG, Apezteguia C, et al. Pandemic 2009 influenza A in Argentina: a study of 337 patients on mechanical ventilation. $\mathrm{Am}$ $J$ Respir Crit Care Med. 2010;182(1):41-48.

11. Chiche L, Forel JM, Roch A, et al. Active cytomegalovirus infection is common in mechanically ventilated medical intensive care unit patients. Crit Care Med. 2009;37(6):1850-1857.

12. Jaber S, Chanques G, Borry J, et al. Cytomegalovirus infection in critically ill patients: associated factors and consequences. Chest. 2005; 127(1):233-241.

13. Papazian L, Thomas P, Bregeon F, et al. Open-lung biopsy in patients with acute respiratory distress syndrome. Anesthesiology. 1998;88(4):935-944.

14. Bousbia S, Papazian L, Saux P, et al. Repertoire of intensive care unit pneumonia microbiota. PLoS One. 2012;7(2):e32486.

15. Mizgerd JP, Skerrett SJ. Animal models of human pneumonia. Am J Physiol Lung Cell Mol Physiol. 2008;294(3):L387-L398.

16. Johanson WG Jr, Seidenfeld JJ, Gomez P, de los SR, Coalson JJ. Bacteriologic diagnosis of nosocomial pneumonia followingprolonged mechanical ventilation. Am Rev Respir Dis. 1988;137(2):259-264.

17. Mall M, Grubb BR, Harkema JR, O’Neal WK, Boucher RC. Increased airway epithelial $\mathrm{Na}+$ absorption produces cystic fibrosis-like lung disease in mice. Nat Med. 2004;10(5):487-493.

18. Gehrig S, Duerr J, Weitnauer M, et al. Lack of neutrophil elastase reduces inflammation, mucus hypersecretion, and emphysema, but not mucus obstruction, in mice with cystic fibrosis-like lung disease. $\mathrm{Am}$ J Respir Crit Care Med. 2014;189(9):1082-1092.
19. Ginsberg HS, Moldawer LL, Sehgal PB, et al. A mouse model for investigating the molecular pathogenesis of adenovirus pneumonia. Proc Natl Acad Sci U S A. 1991;88(5):1651-1655.

20. Kimura K, Miyazaki S, Tateda K, Matsumoto T, Tsujimoto S, Yamaguchi K. Factors affecting the course and severity of transnasally induced Staphylococcus aureus pneumonia in mice. J Med Microbiol. 1999;48(11):1005-1010.

21. Southam DS, Dolovich M, O'Byrne PM, Inman MD. Distribution of intranasal instillations in mice: effects of volume, time, body position, and anesthesia. Am J Physiol Lung Cell Mol Physiol. 2002;282(4): L833-L839.

22. LaForce FM, Boose DS. A radiolabel ratio method for measuring pulmonary clearance of intratracheal bacterial challenges. $J$ Lab Clin Med. 1988;111(2):158-165.

23. Brain JD, Knudson DE, Sorokin SP, Davis MA. Pulmonary distribution of particles given by intratracheal instillation or by aerosol inhalation. Environ Res. 1976;11(1):13-33.

24. Hraiech S, Bregeon F, Brunel JM, et al. Antibacterial efficacy of inhaled squalamine in a rat model of chronic Pseudomonas aeruginosa pneumonia. J Antimicrob Chemother. 2012;67(10):2452-2458.

25. Cash HA, Woods DE, McCullough B, Johanson WG Jr, Bass JA. A rat model of chronic respiratory infection with Pseudomonas aeruginosa. Am Rev Respir Dis. 1979;119(3):453-459.

26. Pedersen SS, Shand GH, Hansen BL, Hansen GN. Induction of experimental chronic Pseudomonas aeruginosa lung infection with P. aeruginosa entrapped in alginate microspheres. APMIS. 1990; 98(3):203-211.

27. McCullers JA, Rehg JE. Lethal synergism between influenza virus and Streptococcus pneumoniae: characterization of a mouse model and the role of platelet-activating factor receptor. J Infect Dis. 2002;186(3):341-350.

28. Morens DM, Taubenberger JK, Fauci AS. Predominant role of bacterial pneumonia as a cause of death in pandemic influenza: implications for pandemic influenza preparedness. J Infect Dis. 2008;198(7):962-970.

29. Shope RE. SWINE INFLUENZA : III. FILTRATION EXPERIMENTS AND ETIOLOGY. J Exp Med. 1931;54(3):373-385.

30. Seki M, Yanagihara K, Higashiyama Y, et al. Immunokinetics in severe pneumonia due to influenza virus and bacteria coinfection in mice. Eur Respir J. 2004;24(1):143-149.

31. LeVine AM, Koeningsknecht V, Stark JM. Decreased pulmonary clearance of S. pneumoniae following influenza A infection in mice. $J$ Virol Methods. 2001;94(1-2):173-186.

32. Cundell DR, Gerard NP, Gerard C, Idanpaan-Heikkila I, Tuomanen EI. Streptococcus pneumoniae anchor to activated human cells by the receptor for platelet-activating factor. Nature. 1995;377(6548):435-438.

33. Ishizuka S, Yamaya M, Suzuki T, et al. Acid exposure stimulates the adherence of Streptococcus pneumoniae to cultured human airway epithelial cells: effects on platelet-activating factor receptor expression. Am J Respir Cell Mol Biol. 2001;24(4):459-468.

34. Kosai K, Seki M, Yanagihara K, et al. Two-dimensional gel electrophoresis analysis in simultaneous influenza pneumonia and bacterial infection in mice. Clin Exp Immunol. 2008;152(2):364-371.

35. Loffler B, Niemann S, Ehrhardt C, et al. Pathogenesis of Staphylococcus aureus necrotizing pneumonia: the role of PVL and an influenza coinfection. Expert Rev Anti Infect Ther. 2013;11(10):1041-1051.

36. Lee MH, Arrecubieta C, Martin FJ, Prince A, Borczuk AC, Lowy FD. A postinfluenza model of Staphylococcus aureus pneumonia. $J$ Infect Dis. 2010;201(4):508-515.

37. Braun LE, Sutter DE, Eichelberger MC, et al. Co-infection of the cotton rat (Sigmodon hispidus) with Staphylococcus aureus and influenza A virus results in synergistic disease. Microb Pathog. 2007;43(5-6): 208-216.

38. Lee LN, Dias P, Han D, et al. A mouse model of lethal synergism between influenza virus and Haemophilus influenzae. Am J Pathol. 2010;176(2):800-811 
39. Loving CL, Brockmeier SL, Vincent AL, Palmer MV, Sacco RE, Nicholson TL. Influenza virus coinfection with Bordetella bronchiseptica enhances bacterial colonization and host responses exacerbating pulmonary lesions. Microb Pathog. 2010;49(5):237-245.

40. Spilker T, Liwienski AA, Lipuma JJ. Identification of Bordetella spp. in respiratory specimens from individuals with cystic fibrosis. Clin Microbiol Infect. 2008;14(5):504-506.

41. Kukavica-Ibrulj I, Hamelin ME, Prince GA, et al. Infection with human metapneumovirus predisposes mice to severe pneumococcal pneumonia. J Virol. 2009;83(3):1341-1349.

42. Yamasaki K, Kawanami T, Yatera K, et al. Significance of anaerobes and oral bacteria in community-acquired pneumonia. PLoS One. 2013;8(5):e63103.

43. Davis CG, Chang K, Osborne D, et al. TLR3 agonist improves survival to secondary pneumonia in a double injury model. J Surg Res. 2013;182(2):270-276.

44. Hibbing ME, Fuqua C, Parsek MR, Peterson SB. Bacterial competition: surviving and thriving in the microbial jungle. Nat Rev Microbiol. 2010;8(1):15-25.

45. Baldan R, Cigana C, Testa F, et al. Adaptation of Pseudomonas aeruginosa in Cystic Fibrosis airways influences virulence of Staphylococcus aureus in vitro and murine models of co-infection. PLoS One. 2014;9(3):e89614.

46. Bittar F, Rolain JM. Detection and accurate identification of new or emerging bacteria in cystic fibrosis patients. Clin Microbiol Infect. 2010;16(7):809-820.

47. Bragonzi A, Farulla I, Paroni M, et al. Modelling co-infection of the cystic fibrosis lung by Pseudomonas aeruginosa and Burkholderia cenocepacia reveals influences on biofilm formation and host response. PLoS One. 2012;7(12):e52330.

48. Vandecandelaere I, Matthijs N, Van NF, et al. Assessment of microbial diversity in biofilms recovered from endotracheal tubes using culture dependent and independent approaches. PLoS One. 2012; 7(6):e38401.

49. Kannangara DW, Thadepalli H, Bach VT, Webb D. Animal model for anaerobic lung abscess. Infect Immun. 1981;31(2):592-597.

50. Kimizuka R, Kato T, Ishihara K, Okuda K. Mixed infections with Porphyromonas gingivalis and Treponema denticola cause excessive inflammatory responses in a mouse pneumonia model compared with monoinfections. Microbes Infect. 2003;5(15):1357-1362.

51. el-Ebiary M, Torres A, Fabregas N, et al. Significance of the isolation of Candida species from respiratory samples in critically ill, nonneutropenic patients. An immediate postmortem histologic study. Am J Respir Crit Care Med. 1997;156(2 Pt 1):583-590.

52. Rello J, Esandi ME, Diaz E, Mariscal D, Gallego M, Valles J. The role of Candida sp isolated from bronchoscopic samples in nonneutropenic patients. Chest. 1998;114(1):146-149.

53. Ricard JD, Roux D. Candida pneumonia in the ICU: myth or reality? Intensive Care Med. 2009;35(9):1500-1502.

54. Azoulay E, Timsit JF, Tafflet M, et al. Candida colonization of the respiratory tract and subsequent pseudomonas ventilator-associated pneumonia. Chest. 2006;129(1):110-117.

55. Roux D, Gaudry S, Khoy-Ear L, et al. Airway fungal colonization compromises the immune system allowing bacterial pneumonia to prevail. Crit Care Med. 2013;41(9):e191-e199.
56. Roux D, Gaudry S, Dreyfuss D, et al. Candida albicans impairs macrophage function and facilitates Pseudomonas aeruginosa pneumonia in rat. Crit Care Med. 2009;37(3):1062-1067.

57. Ader F, Jawhara S, Nseir S, et al. Short term Candida albicans colonization reduces Pseudomonas aeruginosa-related lung injury and bacterial burden in a murine model. Crit Care. 2011;15(3):R150.

58. Zielinski CE, Mele F, Aschenbrenner D, et al. Pathogen-induced human TH17 cells produce IFN-gamma or IL-10 and are regulated by IL-1beta. Nature. 2012;484(7395):514-518.

59. Qureshi MH, Garvy BA, Pomeroy C, Inayat MS, Oakley OR. A murine model of dual infection with cytomegalovirus and Pneumocystis carinii: effects of virus-induced immunomodulation on disease progression. Virus Res. 2005;114(1-2):35-44.

60. Oz HS, Hughes WT, Varilek GW. A rat model for combined Trypanosoma cruzi and Pneumocystis carinii infection. Microb Pathog. 2000;29(6):363-365.

61. Hackam DG, Redelmeier DA. Translation of research evidence from animals to humans. JAMA. 2006;296(14):1731-1732.

62. Pene F, Zuber B, Courtine E, et al. Dendritic cells modulate lung response to Pseudomonas aeruginosa in a murine model of sepsisinduced immune dysfunction. J Immunol. 2008;181(12):8513-8520.

63. Clark CA, Thomas LK, Azghani AO. Inhibition of protein kinase C attenuates Pseudomonas aeruginosa elastase-induced epithelial barrier disruption. Am J Respir Cell Mol Biol. 2011;45(6):1263-1271.

64. Carson JL, Collier AM, Gambling TM, Knowles MR, Boucher RC. Ultrastructure of airway epithelial cell membranes among patients with cystic fibrosis. Hum Pathol. 1990;21(6):640-647.

65. Eutamene H, Theodorou V, Schmidlin F, et al. LPS-induced lung inflammation is linked to increased epithelial permeability: role of MLCK. Eur Respir J. 2005;25(5):789-796.

66. Riffo-Vasquez Y, Coates AR, Page CP, Spina D. Mycobacterium tuberculosis chaperonin 60.1 inhibits leukocyte diapedesis in a murine model of allergic lung inflammation. Am J Respir Cell Mol Biol. 2012;47(2):245-252.

67. Chen ZY, Wang YX, Yang F, Lin Y, Zhou QL, Liao YY. New researches and application progress of commonly used optical molecular imaging technology. Biomed Res Int. 2014;2014:429198.

68. Chang M, Anttonen KP, Cirillo SL, Francis KP, Cirillo JD. Real-time bioluminescence imaging of mixed mycobacterial infections. PLoS One. 2014;9(9):e108341.

69. Stanton TB. A call for antibiotic alternatives research. Trends Microbiol. 2013;21(3):111-113.

70. Ciofi Degli AM, Bernaschi P, Carletti M, et al. An outbreak of extremely drug-resistant Pseudomonas aeruginosa in a tertiary care pediatric hospital in Italy. BMC Infect Dis. 2014;14:494.

71. Hraiech S, Hiblot J, Lafleur J, et al. Inhaled lactonase reduces Pseudomonas aeruginosa quorum sensing and mortality in rat pneumonia. PLoS One. 2014;9(10):e107125.

72. Debarbieux L, Leduc D, Maura D, et al. Bacteriophages can treat and prevent Pseudomonas aeruginosa lung infections. J Infect Dis. 2010;201(7):1096-1104.
Drug Design, Development and Therapy

\section{Publish your work in this journal}

Drug Design, Development and Therapy is an international, peerreviewed open-access journal that spans the spectrum of drug design and development through to clinical applications. Clinical outcomes, patient safety, and programs for the development and effective, safe, and sustained use of medicines are a feature of the journal, which

\section{Dovepress}

has also been accepted for indexing on PubMed Central. The manuscript management system is completely online and includes a very quick and fair peer-review system, which is all easy to use. Visit http://www.dovepress.com/testimonials.php to read real quotes from published authors. 